\title{
RESSONÂNCIA NUCLEAR MAGNÉTICA NOS DISRAFISMOS ESPINAIS
}

\author{
MÁRCIA REGINA DE SOUZA*, JOSÉ PÍNDARO PEREIRA PLESE**, \\ HAMILTON MATUSHITA***, ORILDO CIQUINI***
}

\begin{abstract}
RESUMO - Foi avaliada por ressonância nuclear magnética a incidência de malformações da medula espinal em 38 crianças com espinha bífida. Destas, 22 apresentavam espinha bífida aberta e 16 espinha bífida oculta. Medula presa foi detectada na maioria dos pacientes. Siringomielia foi encontrada em 13 casos. A transição occipitocervical foi estudada em 11 pacientes com meningomielocele e malformação de Chiari foi encontrada em 8 . A análise da casuística permitiu comprovar que existe número significativo de malformações assintomáticas detectadas pela RNM.
\end{abstract}

PALAVRAS-CHAVES: medula espinhal, malformação, espinha bífida, ressonância magnética.

\section{Magnetic resonance imaging of spinal dysraphism}

SUMMARY - We studied by magnetic resonance imaging the incidence of associated malformations in a group of 38 patients with diagnosis of spina bifida followed in our outpatient clinic. Twenty-two children were born with spina aperta and 16 with spina bifida occulta. Tethered cord was found in the majority of patients of both groups. Syringomyelia was found in 13 patients. The craniocervical region was studied in 11 patients with diagnosis of spina bifida aperta; Chiari malformation was found in 8 . The results point to the fact that asymptomatic malformations detected by MRI techniques are frequent in patients with spina bifida.

KEY WORDS: spinal cord, malformation, spina bifida, magnetic resonance imaging.

Espinha bífida (EB) é termo genérico aplicado a grande variedade de malformações que acometem a medula espinal, raízes espinais, seus envoltórios e a coluna vertebral, consequentes a falha da neurulação. Após os estudos de Lemire ${ }^{5}$, este conceito foi ampliado e atualmente engloba grande espectro de malformações que vão desde lesões neurológicas incompatíveis à vida até pequenas alterações somente detectadas por neuroimagem ${ }^{1,3}$. Classicamente as EB são divididas em duas formas clínicas, a espinha bífida aberta (EBA) e a espinha bífida oculta (EBO). Na variedade aberta, o defeito é franco e presente ao nascimento; na oculta a malformação é inaparente e não acompanhada, geralmente, de alterações clínicas ao nascimento. Num passado não muito distante a incidência de outras malformações do sistema nervoso central associadas a EB eram conhecidas através de estudos anatomopatológicos ${ }^{5}$. Com o aperfeiçoamento das técnicas neurorradiológicas e principalmente com o surgimento da ressonância nuclear magnética (RNM), estas têm sido detectadas com maior frequência mesmo em pacientes assintomáticos.

O propósito deste estudo é correlacionar os achados da RNM ao quadro clínico de 38 pacientes com EB seguidos pelo grupo de neurocirurgia pediátrica da Disciplina de Neurocirurgia do Departamento de Neurologia da Faculdade de Medicina de São Paulo.

Disciplina de Neurocirurgia (DNC), Departamento de Neurologia, Faculdade de Medicina da Universidade de São Paulo (FMUSP): *Médica Colaboradora da DNC; **Professor Associado da DNC; ***Médico Assistente do Serviço de Neurologia de Emergência da Divisão de Clínica Neurológica do Hospital das Clínicas da FMUSP. Aceite: 19-outubro-1993.

Dr. José Píndaro Pereira Plese - Clínica Neurológica, Hospital das Clínicas, FMUSP - Caixa Postal 8091 - 01065 970 São Paulo SP - Brasil. 


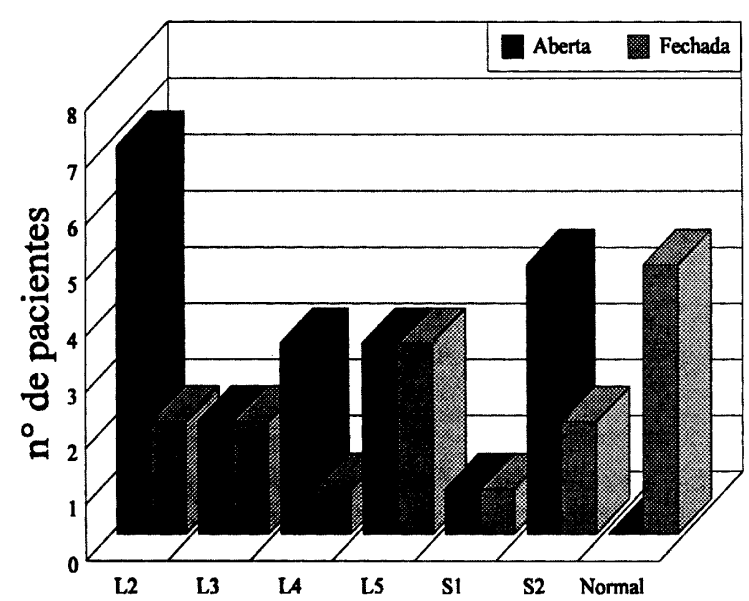

Fig 1. Distribuição do déficit motor (determinado por exame neurológico).

\section{CASUÍSTICA}

A casuística em que se baseia este estudo consta de 38 pacientes com EBA ou EBO, submetidos a avaliação por RNM da coluna vertebral.

Os pacientes foram divididos em dois grupos (A e B). Os do grupo A, em número de 22, tinham EBA já submetida a tratamento neurocirúrgico no período perinatal. A idade em que a RNM foi realizada variou de 2 meses a 13 anos com média de 5,5 anos. Quanto ao sexo, 15 eram femininos e 7 masculinos. Os do grupo $B$, em número de 16, tinham EBO e não foram submetidos a tratamento neurocirúrgico. Destes, 11 eram do sexo feminino e 5 do masculino. Neste grupo a idade variou de 6 meses a 12 anos com média de 5,4 anos.

Todas as crianças foram avaliadas do ponto de vista neurológico, levando-se em conta o nível superior do déficit motor segundo critérios estabelecidos por Plese e Cury ${ }^{7}$. A avaliação da bexiga neurogênica foi feita por critérios clínicos, levando-se em conta principalmente a incontinência e infecções urinárias de repetição. As anormalidades ortopédicas foram caracterizadas fundamentalmente pela presença de escoliose, cifose e alterações plantares.

\section{RESULTADOS}

A análise da Fig 1 mostra que todas as crianças com EBA apresentavam déficit motor: em 7 o nível superior correspondia à raiz L2, em 2 à L3, em 4 à L4, em 4 à L5, em 1 à S1 e em 5 à S2. Mostra ainda que, dentre as com EBO, 5 apresentavam exame neurológico normal, 2 lesões em nível da raiz L2, 2 em L3, 1 em L4, 3 em L5, 1 em S1, $2 \mathrm{em} \mathrm{S2}$.

Hidrocefalia evidente e tratada estava presente em 19 pacientes do grupo A e em nenhum do grupo $\mathrm{B}$. Bexiga neurogênica estava presente em $100 \%$ dos pacientes do grupo A e em $70 \%$ do grupo B; deve-se ressaltar que em duas crianças deste último grupo esse dado não pode ser confirmado, pois eram muito jovens para terem continência vesical. Deformidades dos pés eram patentes em 15 pacientes com EBA e em 8 com EBO.

Em todas as crianças foi pesquisada exaustivamente piora tanto do quadro neurológico como urológico ou ortopédico; sinal e/ou sinais clínicos de deterioração foram detectadores em 11 pacientes do grupo A e em 8 do $B$.

$A$ análise da Fig 2 mostra que as manifestações de piora do quadro ortopédico foram mais frequentes, ocorrendo em $60 \%$ dos casos de EBA e em $40 \%$ dos de EBO. A escoliose foi a manifestação isolada mais frequente, considerando-se os dois grupos. A piora inequívoca dos déficits motores pode ser detectada em 7 pacientes com EBO (70\%) e em nenhum paciente com EBA. Dor lombar com irradiação radicular bilateral foi referida só por um paciente do grupo $B$. Incontinência urinária após o desenvolvimento progressivo de controle esfincteriano foi queixa de dois pacientes com EBO.

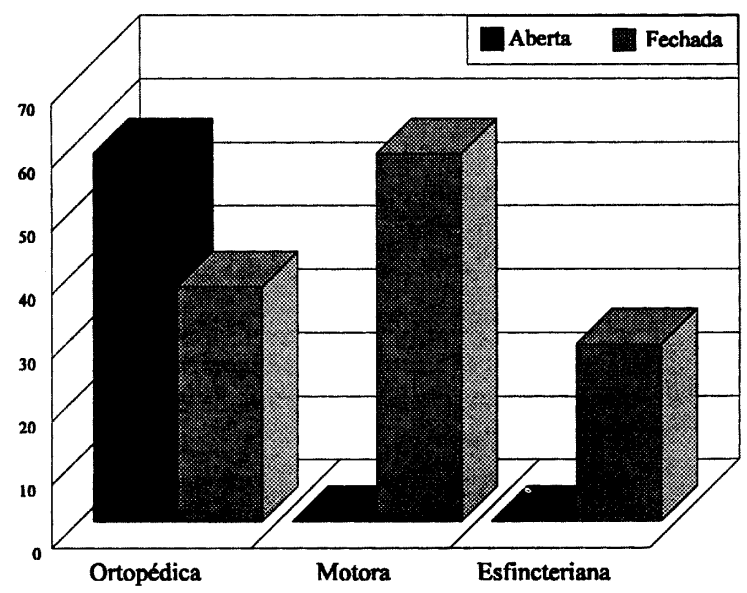

Fig 2. Porcentagem de quadro clínico progressivo relacionado a piora ortopédica, motora e esfincteriana. 


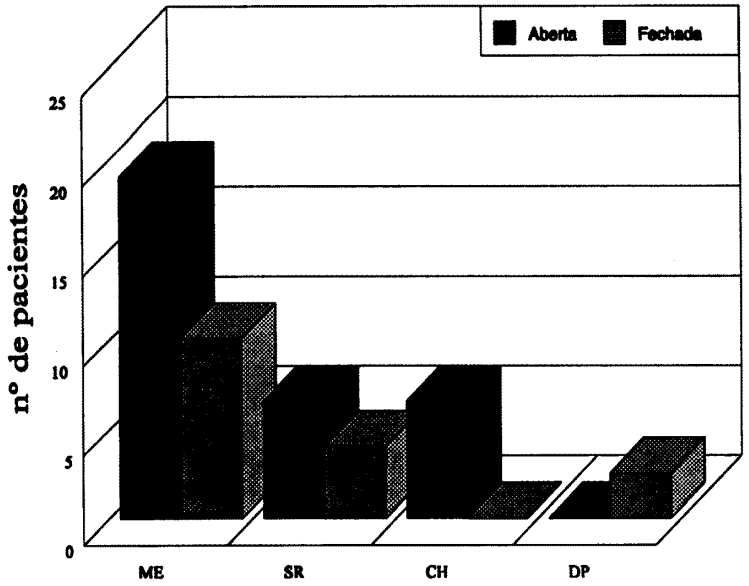

Fig 3. Alteraçōes encontradas na RNM: ME, medula estirada; SE, siringomielia; $\mathrm{CH}$, malformação de Chiari; DP, diplomielia.
A análise da Fig 3 nos mostra que por RNM se identificou em $90 \%$ dos casos do grupo A e em $100 \%$ do B, baixa implantação do cone medular, isto $\epsilon$, que ele se situava em nível inferior a L2-L3. A presença de siringomielina ou hidromielia foi detectada em 8 casos do grupo Ae em 5 do grupo B. Nos 11 pacientes em que se estudou a transição occipitocervical, malformação de Chiari foi encontrada em 8, um deles apenas sendo sintomático. Quatro pacientes do grupo B apresentavam diplomielia, em três tendo sido detectado esporão ósseo.

Aspectos ilustrativos dos achados de RNM são apresentados (Figs 4 a 7).

\section{COMENTÁRIOS}

\section{Com o advento da RNM a} avaliação por neuroimagem das EB tornou-se mais acurada como consequência das imagens de alta resolução que este método permite, além da vantagem de poder ser repetida várias vezes sem que se submeta a criança a radiações ionizantes ${ }^{1,4}$.

Desde a descrição de Garceau² em 1953 da síndrome da medula estirada, muitos estudos têm sido publicados sobre sua fisiopatologia e tratamento ${ }^{5,6.9}$. A maioria dos autores é de opinião que em pacientes com EB, a RNM tem contribuído de maneira significativa para o tratamento desta malformação $0^{1,4,6}$. Publicações recentes enfatizam que por RNM se detecta alta incidência de implantação baixa do cone medular (IBC) e que número significativo destes pacientes não apresenta progressão de seus quadros neurológicos, ortopédicos ou urológicos ${ }^{6}$. A explicação deste fato parece

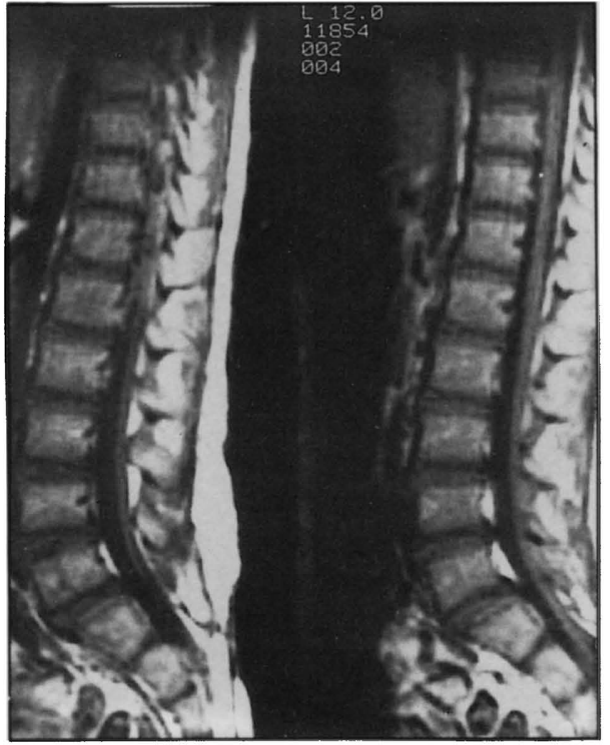

Fig 4.RNM da coluna lombar em corte sagital evidenciando implantação baixa do cone medular em paciente com EBO.

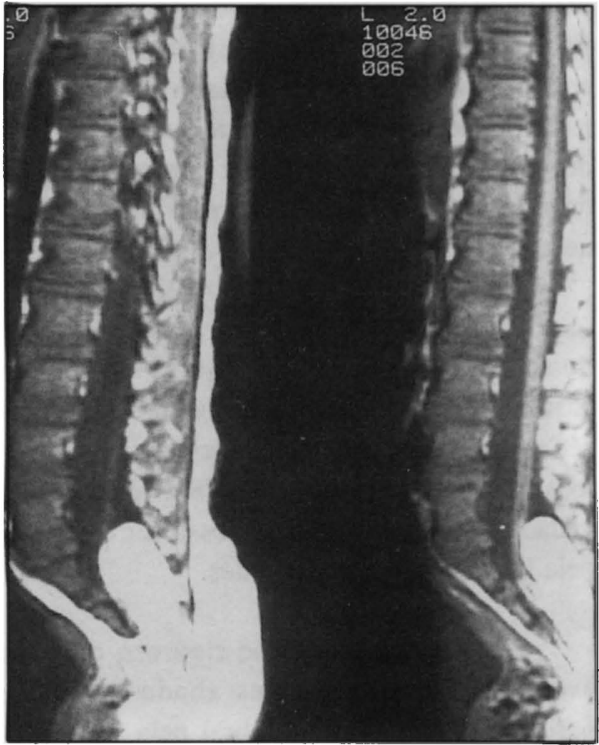

Fig 5. RNM da coluna lombar em corte sagital mostrando lipomielomeningocele e cone medular ao nível da coluna sacra. 


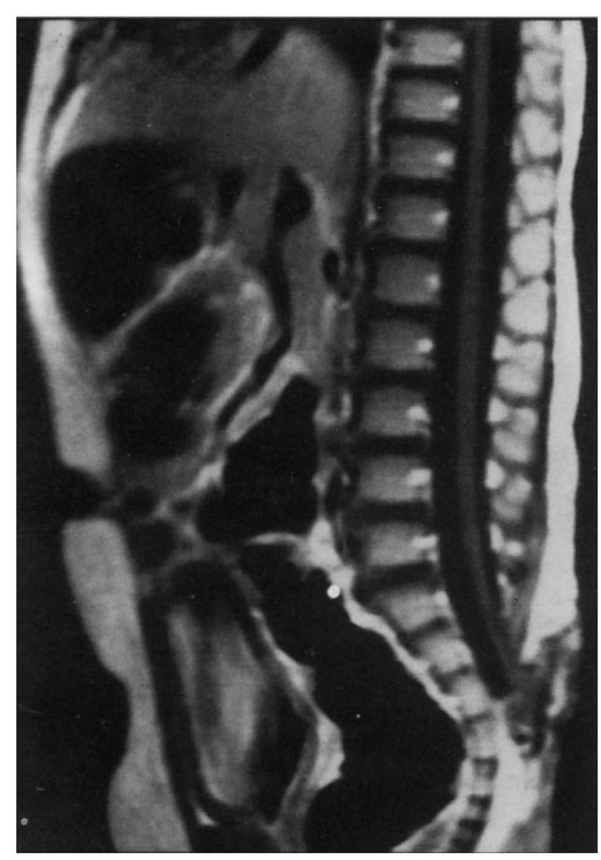

Fig 6. RNM da coluna lombar em corte sagital de paciente operado de EBA mostrando implantação baixa do cone medular.

consequentes a lipomas intrarraqueanos seja devida a alterações metabólicas locais decorrentes de deficiências de perfusão tecidual e de compressão pela massa lipomatosa. Este argumento é usado para explicar as diferentes evoluções clínicas nos pacientes com EBA ou EBO em que foi encontrada IBC. Hirsch e $\mathrm{Kanh}^{3}$ aventam um terceiro mecanismo para esta piora, que seria decorrente da compressão de estruturas neuronais palo lipoma quando da movimentação da coluna vertebral.

A alta incidência de outras malformaçōes do sistema nervoso central observada em nossa casuística é confirmada pela análise de outras séries maiores ${ }^{1,4}$. A ausência de sinais ou sintomas relacionados a estas malformações observada em nossa série talvez possa ser explicada pela baixa idade dos pacientes.

Nossa opinião é que rigoroso controle evolutivo destes pacientes aliado a estudos periódicos da coluna vertebral pela RNM poderão contribuir para melhorar as indicações terapêuticas futuras e prevenir agravamento de defeitos pré-existentes. estar baseada no fator etiológico. Assim, a posição topográfica baixa do cone medular, acarretada por adesões dele à dura-máter após a correção de EBA, teria evolução clínica diferente das causadas por lipomas e pela diastematomielia ${ }^{3}$.

A revisão da casuística de McLone e Naidich $^{6}$ mostra claramente que as crianças com IBC após correção de EBA desenvolveram sinais de deterioração neurológica em alta incidência (53\%), com escoliose progressiva. Este dado também foi observado em nossa casuística.

O mecanismo fisiopatologico dessa escoliose e IBC nos pacientes com EBA corrigida ainda é controverso. A maioria dos autores é de opinião que a IBC agiria em sinergismo com as alterações ósseas existentes, com o desbalanço da musculatura em diferentes graus de função plena, com lesões cordonais posteriores e com a presença de malformações associadas, como a de Chiari e a siringomielia ${ }^{4,7,9}$.

Yamada e Matsumo 9 propõem que a deterioração neurológica dos pacientes com IBC

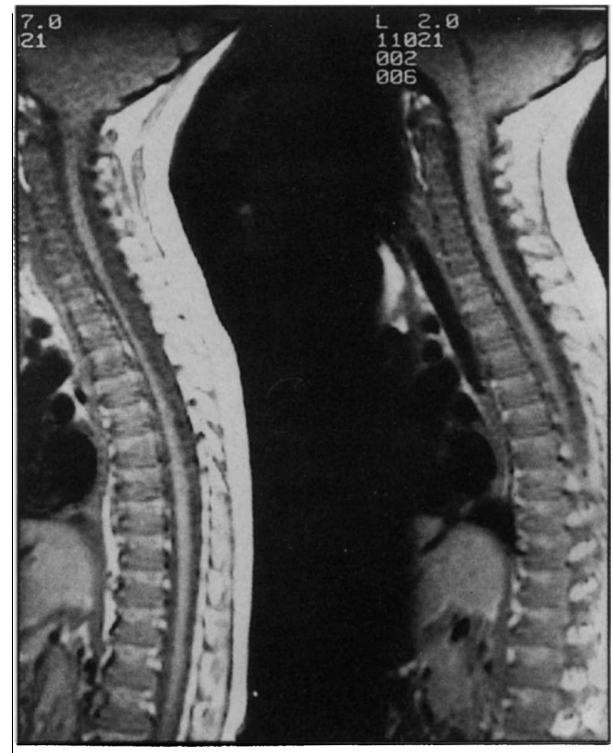

Fig 7. RNM da coluna cervical em corte sagital de paciente operado de EBA evidenciando malformação de Chiari. 


\section{REFERÊNCIAS}

1. Azimullah PC, Smit LM, Rietved-Knol E, Valk J. Malformations of the spinal cord in 53 patients with spina bifida studied by magnetic resonance imaging. Child's Nerv Syst 1991, 7: 53-66.

2. Garceau GJ. The filum terminale syndrome (the cord traction syndrome). J Bone Joint Surg 1953, 35A:711-716.

3. Hirsch JF, Kanh P. Lumbosacral lipomas with spina bifida. Child's Nerv Syst 1988, 4: 354-360.

4. Just M, Schwarz M, Emert JA, Higher HP, Voth A, Pfannentiel P. Magnetic resonance imaging of dysraphic myelodysplasia. Child's Nerv Syst 1988, 4: 149-153.

5. Lemire RJ. Embryology of the central nervous system. In Davis JA, Dobbin J (eds). Scientific foundations of paediatrics. New York: Harper \& Row, 1975.

6. McLone DG, Naidich TP. The tethered spinal cord. In McLaurin RL, Schut L, Venes JL, Epstein F (eds). Pediatric Neurosurgery Ed 2. Philadelphia: Saunders, 1989, p 71-96.

7. Plese JPP, Cury VHSS. Disrafismo espinal. In Diament A, Cypel S (eds). Neurologia Infantil Lefevre. Ed 2. Rio de Janeiro: Atheneu, 1989, p 673-693.

8. Reigel DH. Spina bífida. In McLaurin RL, Schut L, Venes JL, Epstein F (eds). Pediatric Neurosurgery. Ed 2.Philadelphia: Saunders, 1989, p 35-52.

9. Yamada S, Matsumoto S. Tethered cord syndrome versus low-placed conus medullaris in an over distended spinal cord following initial repair for myelodysplasia. Child's Nerv Syst 1990, 6: 264-269. 